Internat. J. Math. \& Math. Sci.

Vol. 24, No. 4 (2000) 251-255

S0161171200003379

(c) Hindawi Publishing Corp.

\title{
LOCAL SUBHOMEOTOPY GROUPS OF BOUNDED SURFACES
}

\author{
DAVID J. SPROWS
}

(Received 2 February 1999 and in revised form 10 May 1999)

\begin{abstract}
Let $M_{n}$ denote the 2-dimensional manifold with boundary obtained by removing the interiors of $n$ disjoint closed disks from a closed 2-manifold $M$ and let $M_{n, r}$ denote the manifold obtained by removing $r$ distinct points from the interior of $M_{n}$. The subhomeotopy group of $M_{n, r}$, denoted $H_{n}\left(M_{n, r}\right)$, is defined to be the group of all isotopy classes (rel $\partial M_{n, r}$ ) of homeomorphisms of $M_{n, r}$ that are the identity on the boundary. In this paper, we use the isotopy classes of various homeomorphisms of $S_{n+1, r}^{2}$ to investigate the subgroup of $H_{n}\left(M_{n, r}\right)$ consisting of those elements that are presented by local homeomorphisms.
\end{abstract}

Keywords and phrases. Local subhomeotopy group, isotopy class, twist homeomorphism, spin homeomorphism.

2000 Mathematics Subject Classification. Primary 57N37.

1. Introduction. Given a 2-dimensional manifold $Y$ with or without boundary, the homeotopy (or mapping class) group of $Y$, denoted $H(Y)$, is defined to be the group of all isotopy classes of homeomorphisms of $Y$ (see [3] or [5] for more details). In the case that $Y$ has a boundary with $n$ boundary components, we define the subhomeotopy group of $Y$, denoted $H_{n}(Y)$, to be the group of all isotopy classes (rel $\partial Y$ ) of homeomorphisms that are the identity on the boundary of $Y$. That is, both the homeomorphisms and the isotopies must be fixed on the boundary of $Y$. Note that the term "local homeomorphism" is used to describe a homeomorphism that is the identity for all values except for those in a "local" region within a given closed disk $D$. This should not be confused with the more standard usage of this term, i.e., a continuous function $f: X \rightarrow Y$ such that each $x$ in $X$ possesses a neighborhood $U$ mapped by $f$ homeomorphically onto a neighborhood $f(U)$ of $x$.

Let $M_{n}$ denote the 2-dimensional manifold with boundary obtained by removing the interiors of $n$ disjoint closed disks from a closed 2-manifold $M$ and let $M_{n, r}$ denote the manifold obtained by removing $r$ distinct points from the interior of $M_{n}$. If we enclose the $n$ disks and $r$ points to be removed from $M$ in a disk $D$, then we say a homeomorphism of $M_{n, r}$ is local provided it is the identity on $M^{\prime}=M-\operatorname{Int}(D)$. For example, if $f$ and $g$ are homeomorphisms of $M_{n, r}$ that represent the same isotopy class in $H_{1}\left(M^{\prime}\right)$, then $f g^{-1}$ is a local homeomorphism. It is the subgroup of $H_{n}\left(M_{n, r}\right)$ consisting of elements represented by local homeomorphisms that is the main concern of this paper.

2. Notation and preliminaries. Let $M$ be a closed manifold and let $D_{1}, \ldots, D_{m}$ be disjoint closed disks in $M$ with centers $p_{1}, \ldots, p_{m}$, where $m=r+n$. Let $D$ be a closed 
disk in $M$ that contains $D_{k}, 1 \leq k \leq m$, in its interior. It will be convenient to consider $D$ as a sphere with the interior of a disk removed. In particular, we will consider the disks $D_{1}, \ldots, D_{m}$ as each having its center on the equator and consider the boundary of $D$ to be the same as the boundary of a disk $D_{m+1}$ that is also centered on the equator. Thus $D \cong S^{2}-\operatorname{Int}\left(D_{m+1}\right)$. Note that under this interpretation, removal of the points $p_{k}, 1 \leq k \leq r$, and the interiors of the disks $D_{k}, r+1 \leq k \leq r+n$, from $M$ transforms $D$ into a space homeomorphic to $S_{n+1, r}^{2}$, i.e., these sphere with $r$ points and $n+1$ open disks removed.

In the following we will make use of three types of homeomorphisms of manifolds with boundary. Twist homeomorphisms are defined in detail in [4, 9]. In essence these are homeomorphisms that are defined on annular neighborhoods of simple closed curves by conjugating the homeomorphism of the annulus $A$ given by $t(r, \theta)=(r, \theta-$ $2 \pi r)$, where $A$ is parametrized by $(r, \theta)$ with $1 \leq r \leq 2$ and $\theta$ a real number $\bmod 2 \pi$.

Given two points $p$ and $q$ contained in the interior of an embedded annulus the corresponding dial homeomorphism essentially "dials" $p$ to $q$ in a counterclockwise direction. Details are given in [7], but again the basic idea is to obtain dial homeomorphisms by suitably conjugating the homeomorphism of $A$ given by $d(r, \theta)=(r, \theta-2 \pi(r-1))$ for $1 \leq r \leq 1.5$ and $d(r, \theta)=(r, \theta-2 \pi(2-r))$ for $1.5 \leq r \leq 2$, where we assume $p$ corresponds to $(3 / 2,0)$ and $q$ to $(3 / 2, \pi)$ in the annulus $A$.

Finally, each boundary component $C$ of a 2-manifold with boundary gives rise to a spin homeomorphism. We define the spin homeomorphism $s$ corresponding to $C$ by letting $e$ be an embedding of the annulus $A$ onto a collar neighborhood of $C$ that sends $r=1 \rightarrow C$ and setting $s=e^{t} e^{-1}$, where $t$ is the twist homeomorphism of $A$.

Note that all three types of homeomorphisms defined above ( $t=$ twist, $d=$ dial, $s=$ spin) are the identity on the boundary of the annulus and so can be extended by the identity from any embedded annulus to the entire manifold containing this embedded annulus.

3. Local homeomorphisms. Let $M$ and $D \cong S^{2}-\operatorname{Int}\left(D_{m+1}\right)$ be as described in the previous section. The first local homeomorphisms we will consider are given by twist homeomorphisms. These twist homeomorphisms will correspond to simple closed curves in $D$, but initially it will be more convenient to describe these curves in $S^{2}$, i.e., the space obtained from $D$ by filling in the disk $D_{m+1}$.

For each $i$ and $j$ with $1 \leq i<j \leq m+1$, let $\alpha_{i j}$ be a simple closed curve in $S^{2}$ that is disjoint from the union of the closed disks $D_{1}, \ldots, D_{m+1}$ and encloses the disks $D_{i}$ and $D_{j}, \ldots, D_{m+1}$. That is, one of the disks bounded by $\alpha_{i j}$ in $S^{2}$ contains $D_{i}$ and $D_{j}, \ldots, D_{m+1}$ and the other disk bounded by $\alpha_{i j}$ contains $D_{1}, \ldots, D_{i-1}$ and $D_{i+1}, \ldots, D_{j-1}$. In addition, assume $\alpha_{i j}$ is below the disks $D_{i+1}, \ldots, D_{j-1}$, i.e., assume the "neck" of $\alpha_{i j}$ is in the southern hemisphere of $S^{2}$. Since $\alpha_{i j}$ is disjoint from the disk $D_{m+1}$, we can consider $\alpha_{i j}$ as a curve in $D \subset M$. Let $a_{i j}$ be the twist homeomorphism of $M$ corresponding to $\alpha_{i j}$.

If we let $M_{n, r}$ be given by $M_{n, r}=M-(F \cup I)$, where $F=\left\{p_{1}, \ldots, p_{r}\right\}$ and $I$ is the union of the interiors of the disks $D_{r+1}, \ldots, D_{m}$, then any homeomorphism $h$ of $M$ that sends $F \cup I$ to itself induces a homeomorphism of $M_{n, r}$. To simplify the notation 
when dealing with such homeomorphisms we will use the same letter denote the given homeomorphism of $M$ and its restriction to $M_{n, r}$.

Note that each $a_{i j}$ represents a local homeomorphism of $M_{n, r}$. Let $H_{n}^{\ell}\left(M_{n, r}\right)$ denote the group of all isotopy classes in $H_{n}\left(M_{n, r}\right)$ that are represented by local homeomorphisms, i.e., homeomorphisms of $M_{n, r}$ that are fixed on $M-D$. These local homeomorphisms must be the identity on the boundary component of each of the disks $D_{r+1}, \ldots, D_{m+1}$, but do not necessarily induce the identity map on $F$ if we fill in each of the points $p_{1}, \ldots, p_{r}$.

In order to deal with local homeomorphisms that may permute the points of $F$, we introduce the following class of dial homeomorphisms. As was the case with the homeomorphisms $a_{i j}$, it will be convenient to first define these dial homeomorphisms on $S^{2}$.

For each pair of adjacent points $p_{i}$ and $p_{i+1}, 1 \leq i<r$, embed an annulus about a simple closed curve passing through $p_{i}$ and $p_{i+1}$. Since each of the disks $D_{j}, 1 \leq$ $j \leq m+1$, is on the equator of $S^{2}$, we can assume that this annulus is disjoint from all of disks $D_{k}$ with $k \neq i, i+1$ and that the embedding takes the segments $\theta=0$ (respectively, $\theta=\pi$ ) to a section of the equator containing $p_{i+1}$ (respectively, $p_{i}$ ). Define $d_{i}$ to be the dial homeomorphism corresponding to $p_{i}$ and $p_{i+1}$. As was the case with the $a_{i j}$, each $d_{i}$ can be considered a homeomorphism of $M_{n, r}$.

Next let $C_{i}$ denote the boundary component of the disk $D_{i}$ for $r+1 \leq r \leq m+1$ and let $s_{i}$ be the corresponding spin homeomorphism.

Given a local homeomorphism $h$ of $M_{n, r}$ we use $\bar{h}$ to denote the equivalence class of $h$ in $H_{n}\left(M_{n, r}\right)$. Note that each local homeomorphism is the identity on each $C_{i}$ for $r+1 \leq r \leq m+1$, but the isotopies needed to determine the equivalence classes in $H_{n}\left(M_{n, r}\right)$ are required to be the identity only on $C_{i}$ for $r+1 \leq i \leq m$.

The following theorem shows that every element in $H_{n}^{\ell}\left(M_{n, r}\right)$ can be expressed in terms of equivalence classes of twists, dials and spins.

THEOREM 3.1. $H_{n}^{\ell}\left(M_{n, r}\right)$ is generated by $T \cup L \cup S$, where

$$
T=\cup_{k=2}^{m+1}\left\{\bar{a}_{i k}: 1 \leq i<k\right\}, \quad L=\left\{\bar{d}_{i}: 1 \leq i<r\right\}, \quad S=\left\{\bar{s}_{i}: r+1 \leq i \leq m+1\right\} .
$$

Moreover, the "commutator" relations among these generators are given as follows:

1. (1) If $p<q$ and $i>t$, then

$$
\bar{a}_{i p} \bar{a}_{t q} \bar{a}_{i p}^{-1}=\left(\bar{a}_{i q} \bar{b}\right) \bar{a}_{t q}\left(\bar{a}_{i q} \bar{b}\right)^{-1}, \quad \text { where } \bar{b}=\bar{a}_{p q} \cdots \bar{a}_{q-1, q} .
$$

(2) If $p<q$ and $i<t<p$, then

$$
\bar{a}_{i p} \bar{a}_{t q} \bar{a}_{i p}^{-1}=\left(\bar{b} \bar{a}_{i q}\right) \bar{a}_{t q}\left(\bar{b} \bar{a}_{i q}\right)^{-1} .
$$

(3) $\bar{a}_{i p} \bar{a}_{t q} \bar{a}_{i p}^{-1}=\bar{a}_{t q}$ in all remaining cases.

2. (1) $\bar{d}_{i} \bar{d}_{k} \bar{d}_{i}^{-1}=\bar{d}_{k}^{-1} \bar{d}_{i} \bar{d}_{k}$, if $|i-k|=1$.

(2) $\bar{d}_{i} \bar{d}_{k} \bar{d}_{i}^{-1}=\bar{d}_{k}$, if $|i-k| \geq 2$.

3. (1) $\bar{d}_{p} \bar{a}_{j k} \bar{d}_{p}^{-1}=\bar{a}_{j+1, k}$, if $j<k-1$ and $p=j$.

(2) $\bar{d}_{p} \bar{a}_{j k} \bar{d}_{p}^{-1}=\bar{a}_{j k}^{-1} \bar{a}_{j-1, k} \bar{a}_{j k}^{-1}$, if $p=j-1$.

(3) $\bar{d}_{p} \bar{a}_{j k} \bar{d}_{p}^{-1}=\bar{a}_{j, k-1} \bar{a}_{j k}^{-1} \bar{a}_{k-1, k}$, if $k=r$ and $j<k-1$ and $p=r-1$.

(4) $\bar{d}_{p} \bar{a}_{j k} \bar{d}_{p}^{-1}=\bar{a}_{j, k-1} \bar{a}_{j, k+1} \bar{a}_{j k}^{-1}\left(\bar{a}_{k-1, k+1} \bar{a}_{k, k+1} \bar{a}_{k+1, k+2}^{-1} \bar{a}_{k-1, k}^{-1}\right)$, if $p=k-1$ and $j<k-1$. 
(5) $\bar{d}_{p} \bar{a}_{j k} \bar{d}_{p}^{-1}=\bar{a}_{j k}$ for all remaining choices of $j, k, p$.

4. (1) $\bar{s}_{i} \bar{c} \bar{s}_{i}^{-1}=\bar{c}$ any generator $\bar{c}$.

The relations given above are expressed in the form that naturally occurs when deriving these relations in $H_{n}^{\ell}\left(M_{n, r}\right)$, but note that each of these relations can be written with the commutator of the given generators on the left by multiplying through by the inverse of the appropriate generator.

Proof OF TheOrem 3.1. Let $G$ be the subgroup of $H_{n}^{\ell}\left(M_{n, r}\right)$ consisting of all those elements that are represented by local homeomorphisms of $M_{n, r}$ that induce the identity on the set $F$ when we fill in the points $p_{1}, \ldots, p_{r}$. The inclusion map gives rise to the short exact sequence (1) $1 \rightarrow G \rightarrow H_{n}^{\ell}\left(M_{n, r}\right) \rightarrow B \rightarrow 1$, where $B$ is isomorphic to the symmetric group $S_{p}$. Since $S_{p}$ is generated by the set of all transpositions of the form $(i, i+1)$, it follows that $L$ is a generating set for $B$.

To find a set of generators for $G$ we first consider the correspondence between local homeomorphisms of $M_{n, r}$ and homeomorphisms of $S_{n+1, r}^{2}$ that is obtained by viewing $D$ as $S^{2}-\operatorname{Int}\left(D_{m+1}\right)$. In particular, if we let $G^{\prime}$ denote the subgroup of $H_{n+1}\left(S_{n+1, r}^{2}\right)$ consisting of all elements that are represented by homeomorphisms that induce the identity on $F$, then there is a natural homeomorphism from $G^{\prime}$ onto $G$ that sends the isotopy class (rel $\partial S_{n+1, r}^{2}$ ) of a homeomorphism $h$ in $G^{\prime}$, denoted $h^{\prime}$, to the isotopy class (rel $\partial M_{n, r}$ ) of $h$ in $G$, denoted $\bar{h}$. Note that since this homeomorphism is onto, any generating set for $G^{\prime}$ gives rise to a generating set (with possible redundancies) for $G$.

Let $I$ (respectively, $I^{\prime}$ ) denote the subgroup of $G$ (respectively, $G^{\prime}$ ) consisting of those elements that are represented by homeomorphism that are isotopic to the identity. That is, a non-trivial element of $I$ (respectively, $I^{\prime}$ ) is isotopic to the identity, but not by an isotopy that is fixed on $\partial M_{n, r}$ (respectively, $\partial S_{n+1, r}^{2}$ ).

The inclusion map induces a short exact sequence (2) $1 \rightarrow I^{\prime} \rightarrow G^{\prime} \stackrel{\sigma}{\rightarrow} H^{\prime} \rightarrow 1$, where $H^{\prime}$ is obtained by identifying two elements of $G^{\prime}$ provided they are isotopic. That is, $\sigma\left(g^{\prime}\right)=\sigma\left(h^{\prime}\right)$ provided $g$ is isotopic to $h$, where the isotopy is not required to be fixed on the boundary.

Since rotations are essentially the only isotopies of the circle that start and end with the identity (see [8]), a generating set for $I^{\prime}$ is given by any set of homeomorphisms of $S_{n+1, r}^{2}$ that give all possible rotations of each $c_{i}, r+1 \leq r \leq m+1$. In particular, $I^{\prime}$ is generated by the set $S^{\prime}=\left\{s_{i}^{\prime}: r+1 \leq i \leq m+1\right\}$.

A generating set for $H^{\prime}$ can be obtained by making use of [6, Theorem 4.1] and the fact that all the homeomorphisms and isotopies given in this theorem can be taken to be fixed on closed disks that contain each of the points involved in the fixed point set. In particular, if we take $\left\{p_{1}, \ldots, p_{m+1}\right\}$ as the fixed point set and use $D_{r+1}, \ldots, D_{m+1}$ as the corresponding disks about the points $p_{r+1}, \ldots, p_{m+1}$, then it follows that $H^{\prime}$ is generated by the set $T^{\prime}$ consisting of those elements that are represented by the twist homeomorphisms $a_{i k}, 1 \leq i<k, k=2, \ldots, m+1$.

Using the short exact sequence (2), we have that $G^{\prime}$ is generated by $T^{\prime} \cup S^{\prime}$. Using the short exact sequence (1), the relationship between $G^{\prime}$ and $G$, and the fact that $B$ is generated by $L$, we have that $G$ is generated by $T \cup L \cup S$.

The relations given in 2(1) and 2(2) are obtained by making use of the corresponding 
relations that hold for the transpositions $(i, i+1)$ is the symmetric group $S_{r}$ (see [1]). The relations given in 4 follow from the fact that the support of each $s_{i}$ can be taken to be disjoint from the supports of the dial and twist homeomorphisms.

The relations in 1 and 3 are determined with the help of two properties of twist homeomorphisms. One useful property is that if $\alpha$ and $\beta$ are simple closed curves in the interior of $M_{n, r}$ and $\alpha$ is isotopic to $\beta\left(\right.$ rel $\partial M_{n, r}$ ), then the twist homeomorphisms corresponding to these curves are isotopic (rel $\left.\partial M_{n, r}\right)$. Another useful property deals with the twist homeomorphism corresponding to $h(\alpha)$, where $h$ is a homeomorphism of $M_{n, r}$ and $\alpha$ is a simple closed curve in the interior of $M_{n, r}$. If we let $a$ denote the twist homeomorphism corresponding to $\alpha$, then the twist homeomorphism given by $h(\alpha)$ is isotopic $h a h^{-1}$. Moreover, if $h$ is fixed on a boundary component $C_{i}$, then we can assume this isotopy also leaves the boundary component of $C_{i}$ fixed. Using these two properties of twist homeomorphisms, together with the techniques described in [6] establishes the relations in 1 and 3 and hence completes the proof of the theorem.

Usually the relations in the theorem do not yield a recognizable group. A description of the group obtained for various values of $m$ and $n$ in the case when $M=S^{2}$ can be found in [6, 7]. Note that, depending on the choice of manifold $M$ and the value of $n$, it may be possible to reduce the size of the generating set given in the theorem. For example, if $M=S^{2}$, then the homeomorphism $s_{m+1}$ is isotopic (rel $\partial M_{n, r}$ ) to the identity, so that the generator $\bar{s}_{m+1}$ is redundant in this case. Similarly, the value of $n$ has an impact on the relations that hold among these generators. In particular, if $n \geq 3$, then relations such as those discussed in $[2,8]$ will hold in $H^{\ell}\left(M_{n, r}\right)$.

\section{REFERENCES}

[1] H. S. M. Coxeter and W. O. J. Moser, Generators and Relations for Discrete Groups, SpringerVerlag, Berlin, 1965. MR 30\#4818. Zbl 133.28002.

[2] D. L. Johnson, Homeomorphisms of a surface which act trivially on homology, Proc. Amer. Math. Soc. 75 (1979), no. 1, 119-125. MR 80h:57008. Zbl 407.57003.

[3] J. P. Lee, Homeotopy groups of orientable 2-manifolds, Fund. Math. 77 (1972), no. 2, 115124. MR 47\#9625. Zbl 241.57020.

[4] W. B. R. Lickorish, A finite set of generators for the homeotopy group of a 2-manifold, Proc. Cambridge Philos. Soc. 60 (1964), 769-778. MR 30\#1500. Zbl 131.20801.

[5] G. S. McCarty, Jr., Homeotopy groups, Trans. Amer. Math. Soc. 106 (1963), 293-304. MR 26\#3062. Zbl 113.17201.

[6] D. J. Sprows, Subhomeotopy groups of the 2-sphere with $n$ holes, Fund. Math. 108 (1980), no. 1, 1-5. MR 81m:57026. Zbl 432.57002.

[7] _ Homeotopy groups of punctured spheres with holes, Fund. Math. 115 (1983), no. 3, 207-212. MR 85c:57037. Zbl 521.57007.

[8] _ A note on the presentations of isotopy groups of bounded 2-manifolds, Kumamoto J. Math. 2 (1989), 28-31. MR 90j:57001. Zbl 684.57007.

[9] H. Zieschang, E. Vogt, and H. Coldewey, Surfaces and Planar Discontinuous Groups, Lecture Notes in Mathematics, vol. 835, Springer, Berlin, 1980. MR 82h:57002. Zbl 438.57001.

DAVID J. SPROWS: DEPARTMENT OF MATHEMATICAL SCIENCES, VILLANOVA UNIVERSITY, VILLANOVA, PA 19085, USA

E-mail address: david.sprows@vi11 anova.edu 


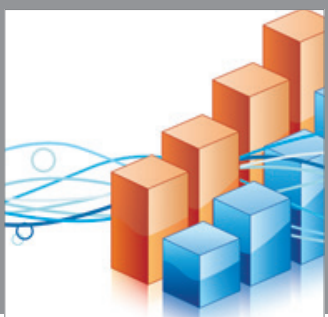

Advances in

Operations Research

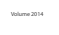

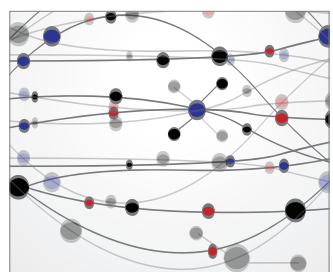

\section{The Scientific} World Journal
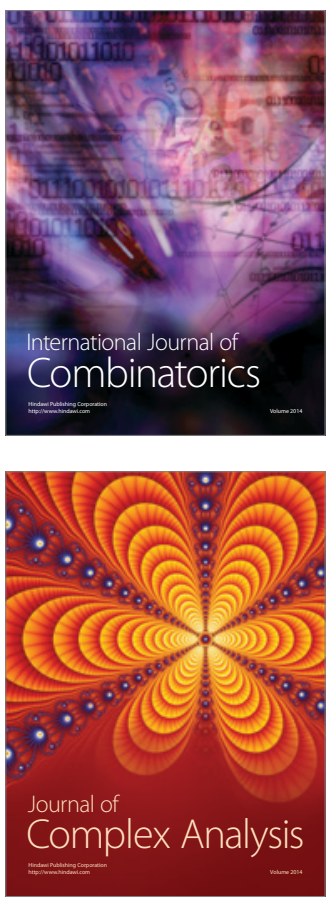

International Journal of

Mathematics and

Mathematical

Sciences
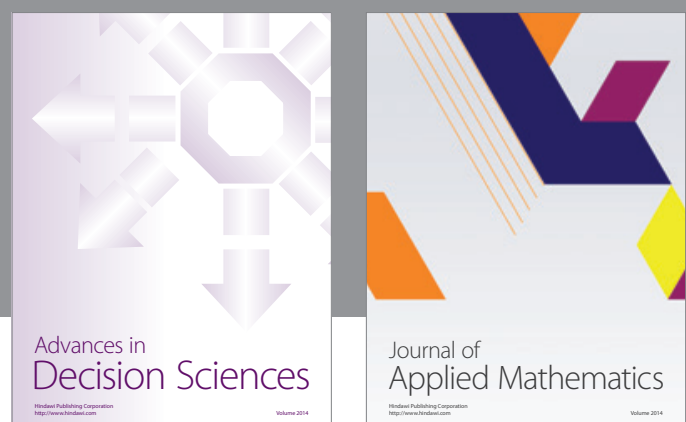

Journal of

Applied Mathematics
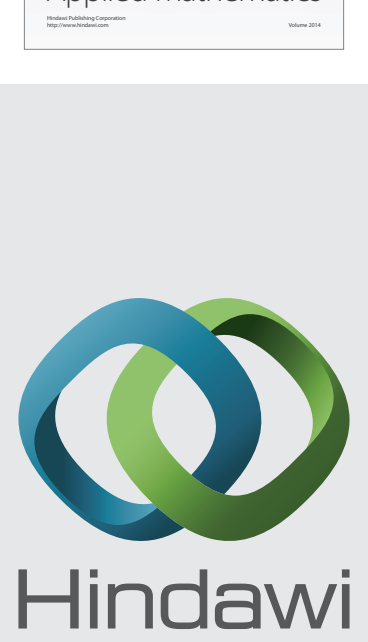

Submit your manuscripts at http://www.hindawi.com
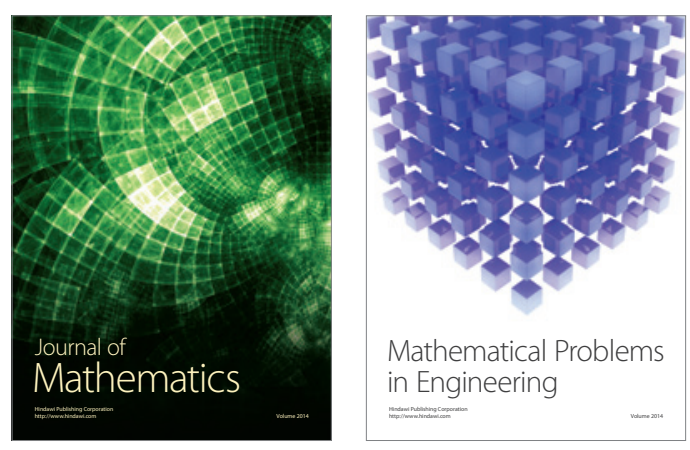

Mathematical Problems in Engineering
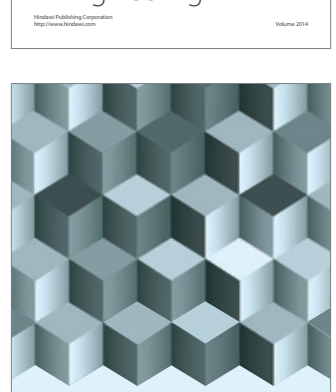

Journal of

Function Spaces
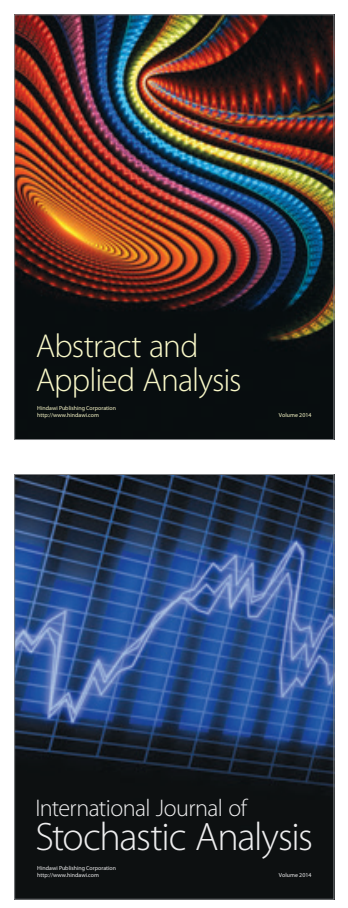

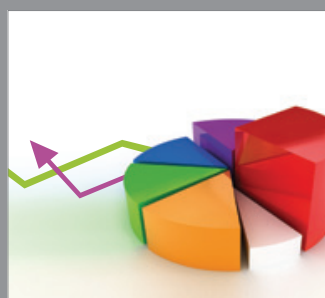

ournal of

Probability and Statistics

Promensencen
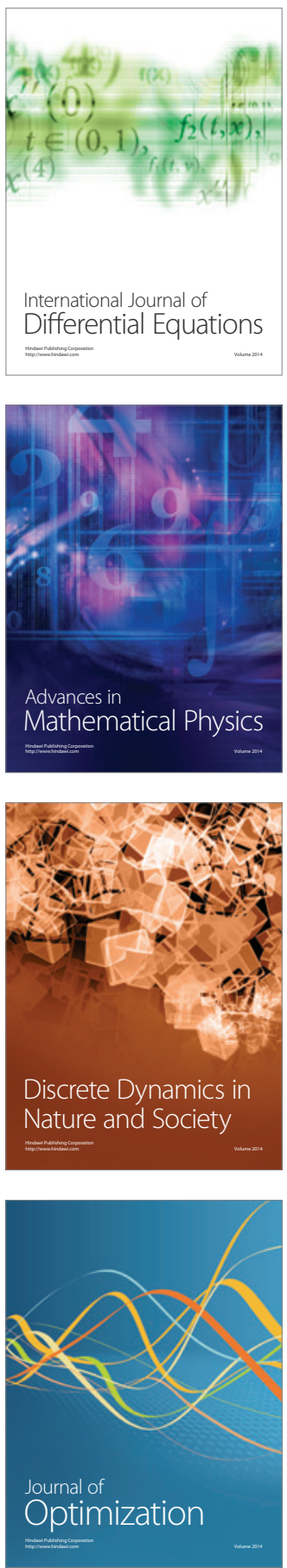